This document is confidential and is proprietary to the American Chemical Society and its authors. Do not copy or disclose without written permission. If you have received this item in error, notify the sender and delete all copies.

\title{
Suppression of MTHFD2 in MCF7 breast cancer cells increases glycolysis, dependency on exogenous glycine and sensitivity to folate depletion
}

\begin{tabular}{|r|l|}
\hline Journal: & Journal of Proteome Research \\
\hline Manuscript ID & pr-2016-00188a.R2 \\
\hline Danuscript Type: & Article \\
\hline Complete List of Authors: & n/a \\
\hline & $\begin{array}{l}\text { Koufaris, Costas; CING, } \\
\text { Gallage, Suchira; Imperial College, Biological Chemistry } \\
\text { Yang, Tianlai; Imperial College, Biological Chemistry } \\
\text { Lau, Chung-Ho; Imperial College, Biological Chemistry } \\
\text { Valbuena, Gabriel; Imperial College, Biological Chemistry } \\
\text { Keun, Hector; Imperial College, Biological Chemistry }\end{array}$ \\
\hline
\end{tabular}




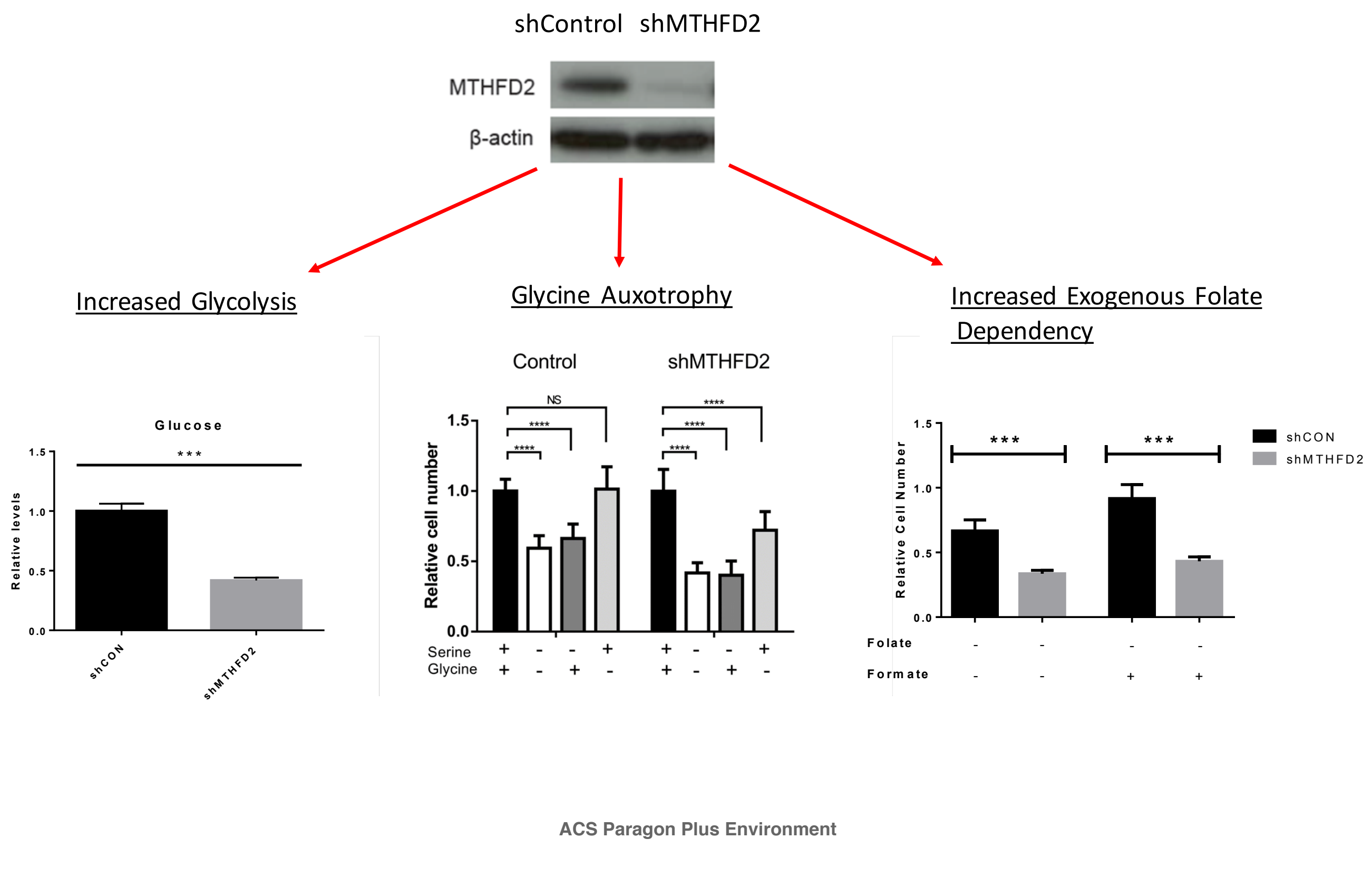




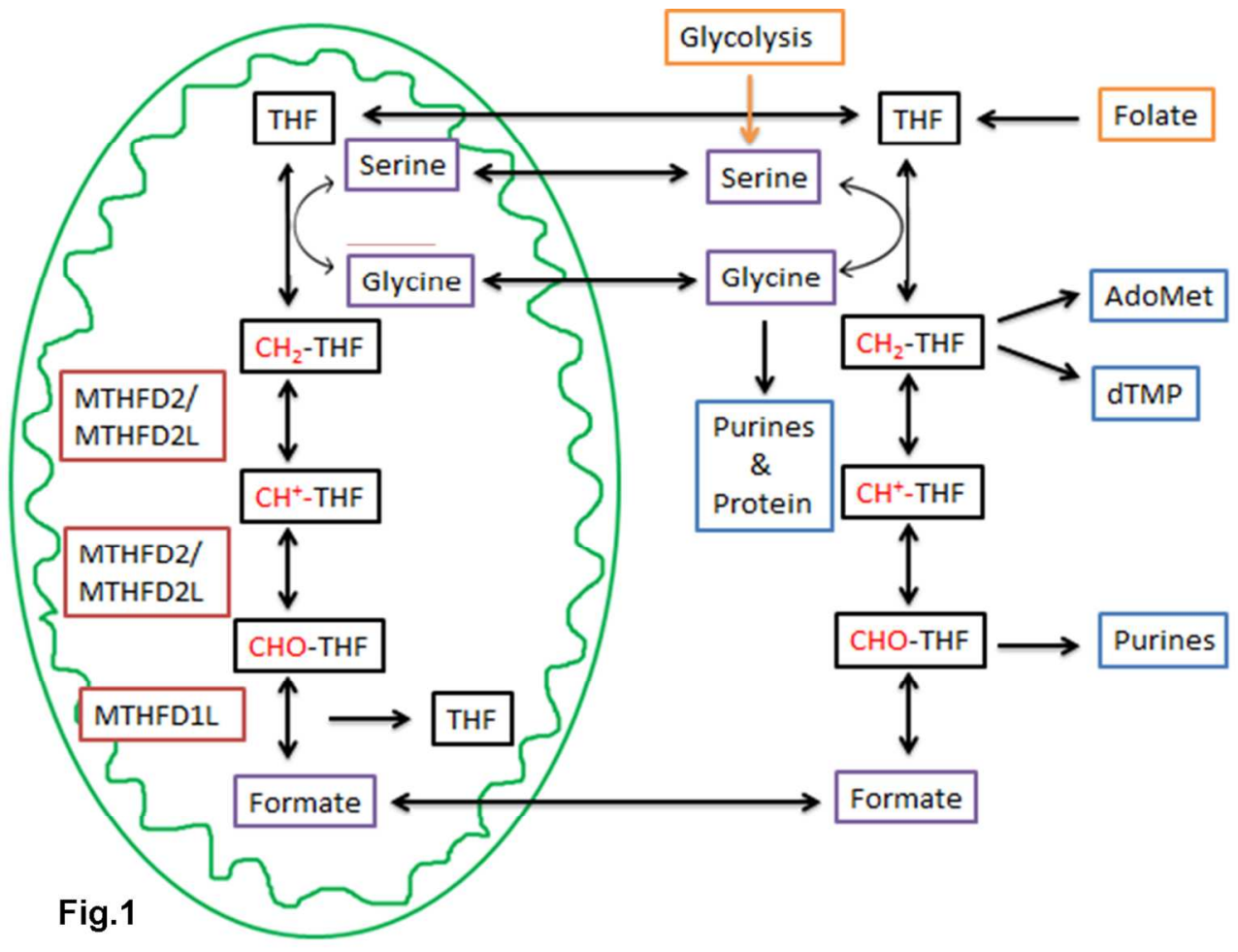

Fig1

$233 \times 174 \mathrm{~mm}(150 \times 150$ DPI $)$ 
A

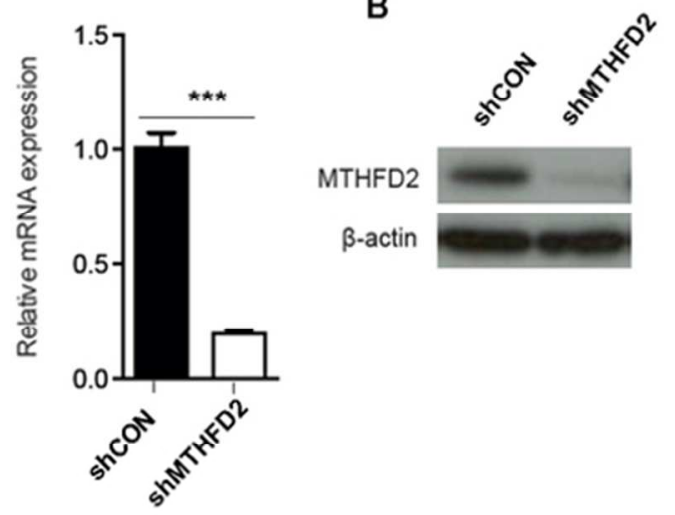

C

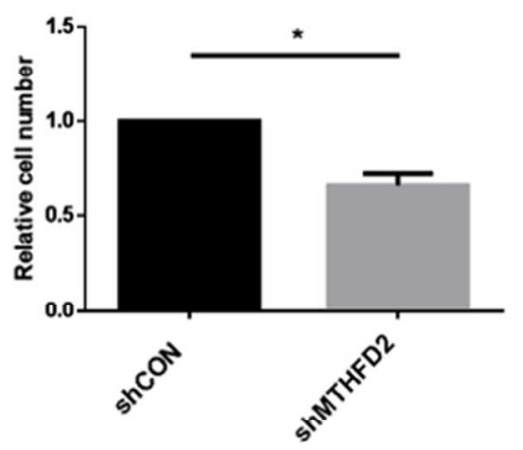

Fig.2

Fig2

$248 \times 108 \mathrm{~mm}(73 \times 74$ DPI $)$ 


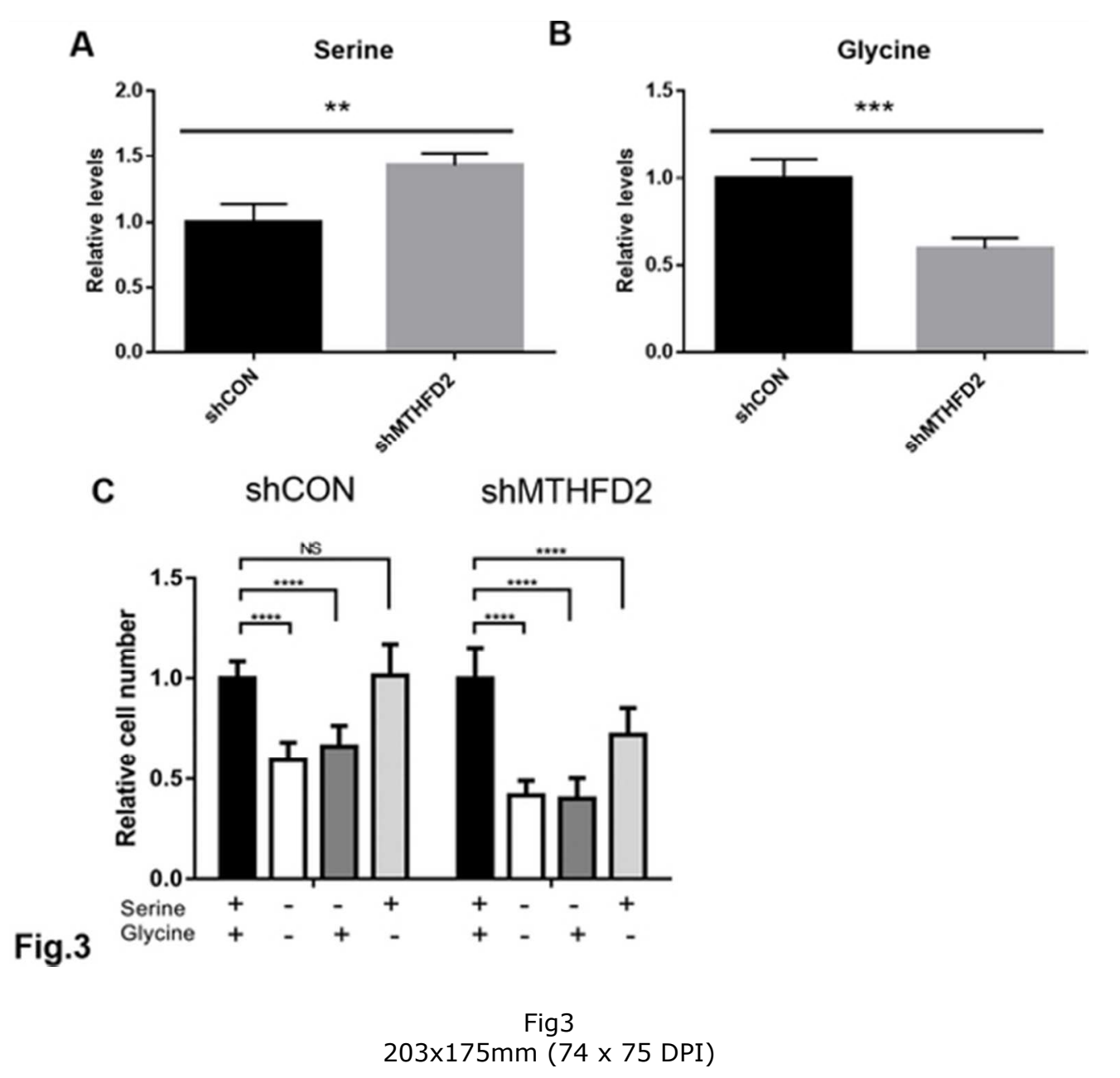


Fig4 $182 \times 93 \mathrm{~mm}(150 \times 150 \mathrm{DPI})$

Fig.4
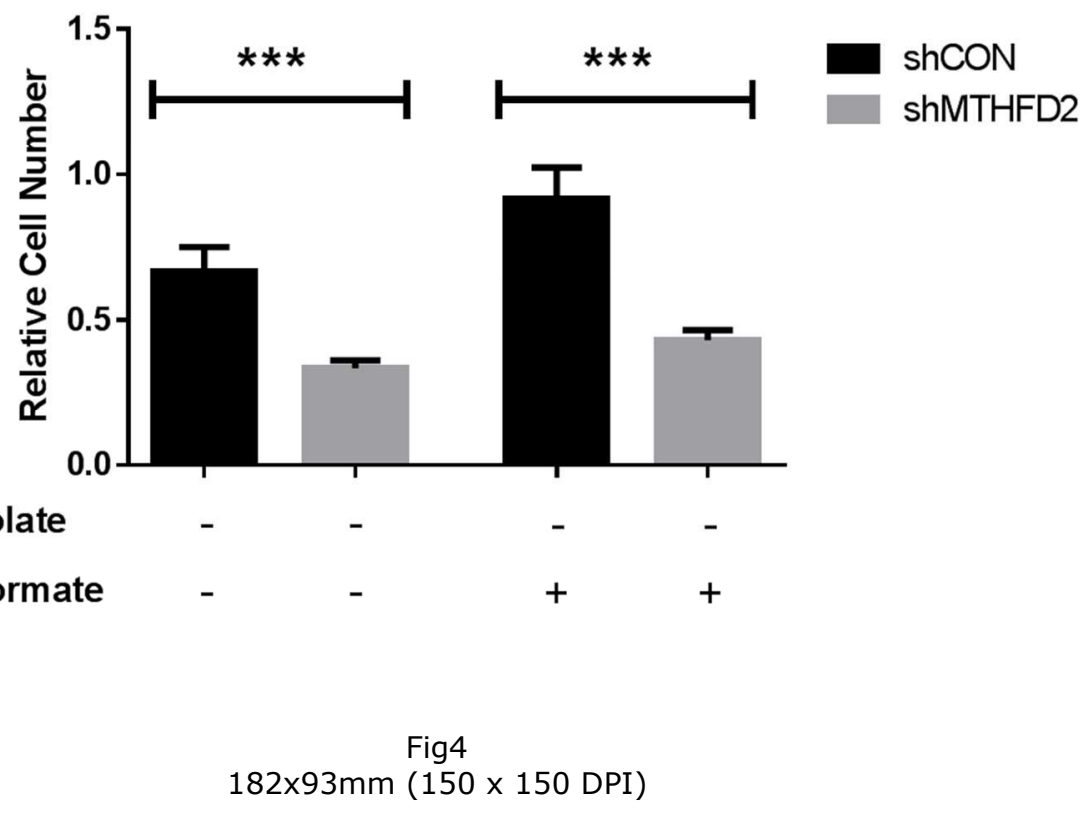
A

1

2

3

3

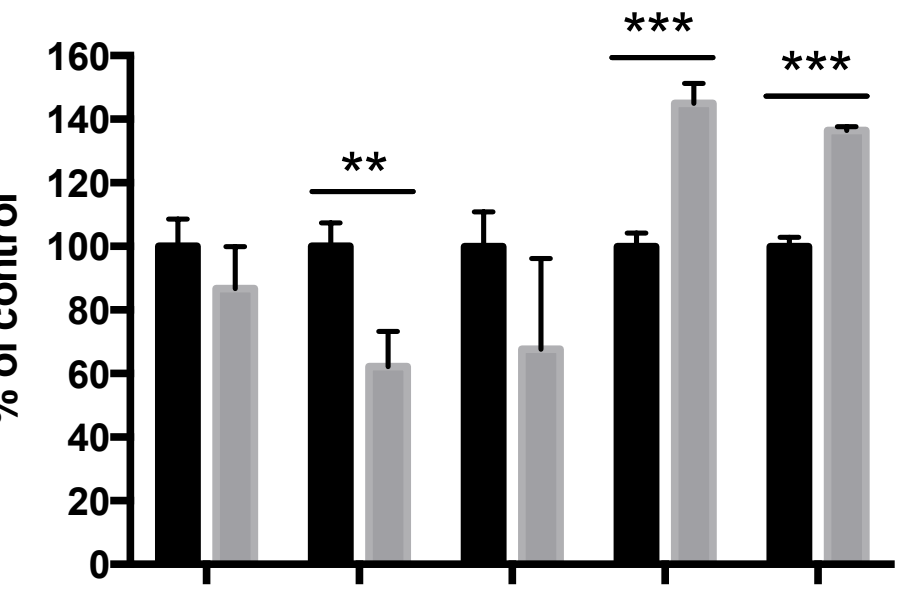

15
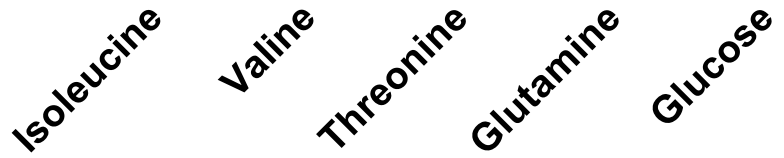

19

20

21

22

23

24

25

26

27

27

20

B

3.

3 전

330

34

35

36

37

38

39

40

41

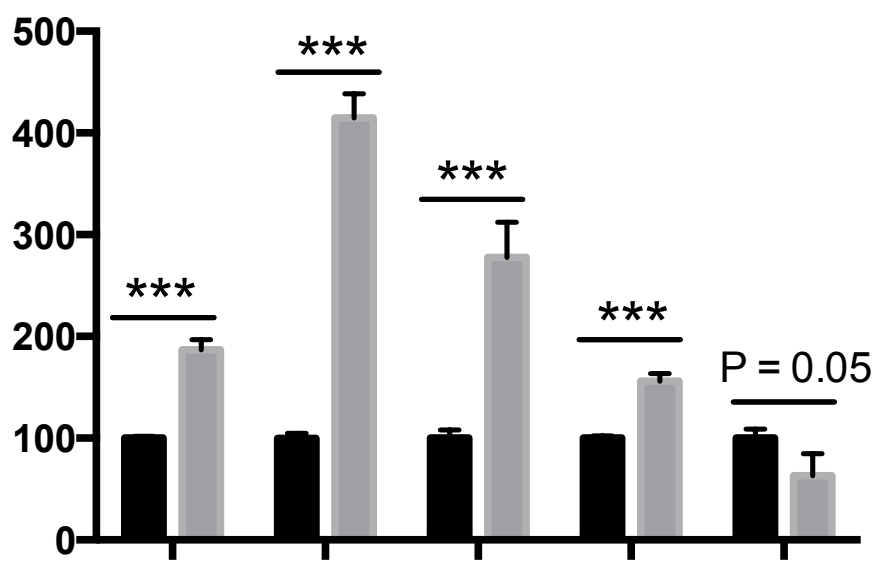

42
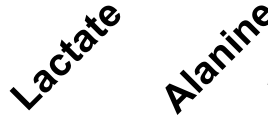

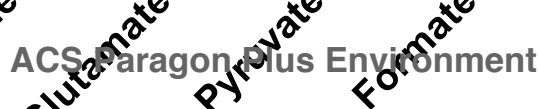

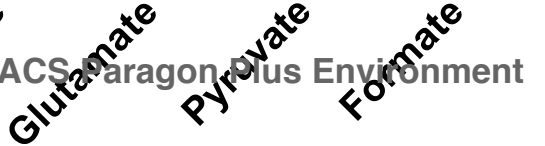


1

2

3

4

5

6

7

8

9

10

11

12

13

14

15

16

17

18

19

20

21

22

23

24

25

26

27

28

29

30

31

32

33

34

35

36

37

38

39

40

41

42

43

44

45

46

47

48

49

50

51

52

53

54

55

56

57

58

59

60
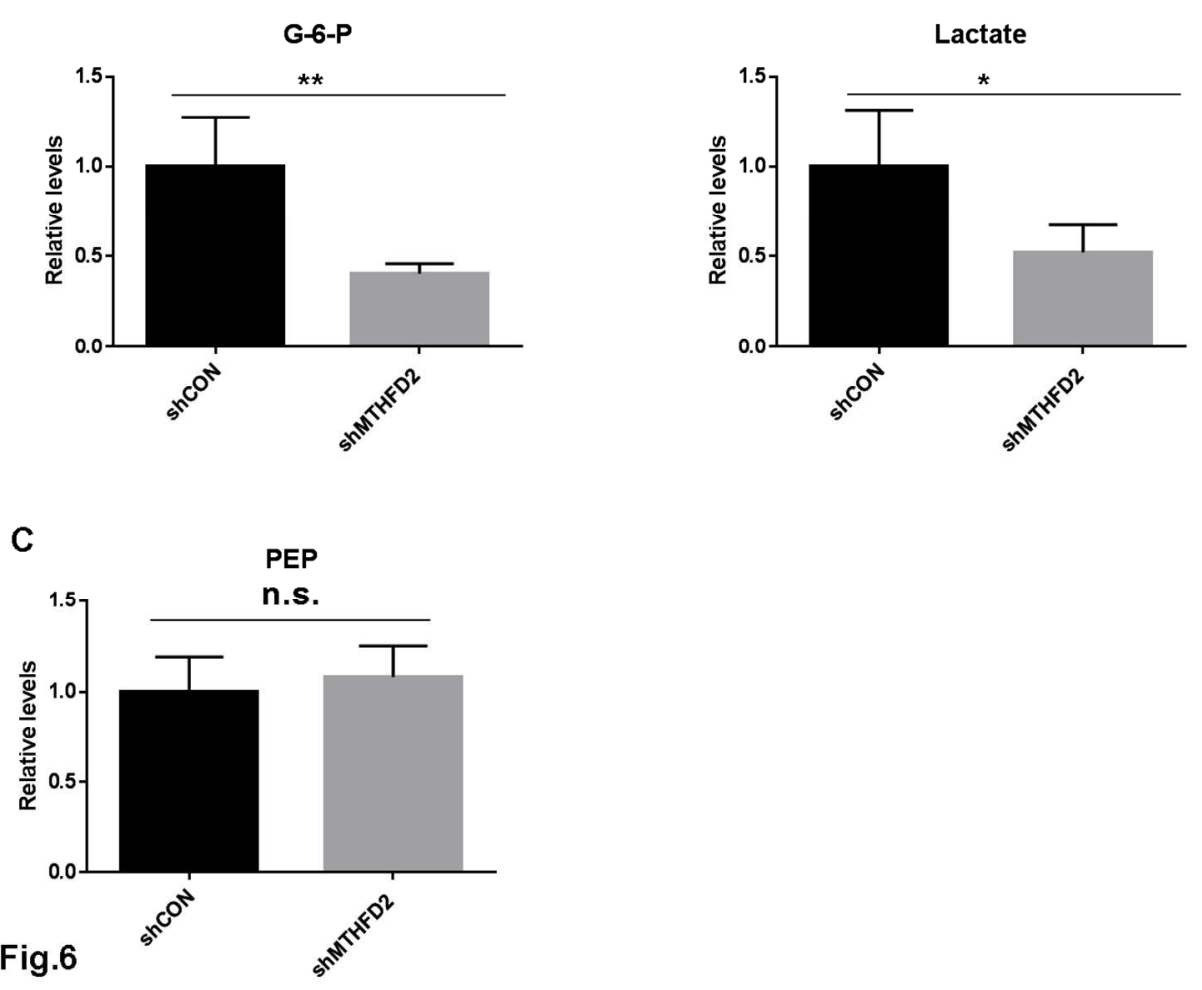

$242 \times 190 m m(150 \times 150$ DPI $)$ 


\section{Suppression of MTHFD2 in MCF-7 breast cancer cells increases glycolysis, dependency on exogenous glycine and sensitivity to folate depletion}

Costas Koufaris ${ }^{1,2 \$}$, Suchira Gallage ${ }^{1 \$}$, Tianlai Yang ${ }^{1}$, Chung-Ho Lau ${ }^{1}$, Gabriel N Valbuena $^{1}$, Hector C. Keun ${ }^{1 *}$

${ }^{1}$ Division of Cancer, Department of Surgery and Cancer, Imperial College London, Hammersmith Hospital, London, UK; ${ }^{2}$ Department of Cytogenetics and Genomics, Cyprus Institute of Neurology and Genetics, Nicosia, Cyprus

* Corresponding author: Dr HC Keun, Division of Cancer, Department of Surgery and Cancer, Imperial College London, Institute of Reproductive and Developmental Biology, Hammersmith Hospital, London W12 0NN, UK.

E-mail: h.keun@imperial.ac.uk; tel +44 (0)20 75943161

Keywords: MTHFD2, cancer, MCF-7, shRNA, metabolomics, folate, glycine, glycolysis

${ }^{\$}$ Indicates that both authors have contributed equally to this manuscript 


\begin{abstract}
Methylenetetrahydrofolate dehydrogenase $(\mathrm{NAD}(\mathrm{P})+\quad$ dependent $) \quad 2$, methenyltetrahydrofolate cyclohydrolase (MTHFD2) is a mitochondrial enzyme involved in folate metabolism. A number of recent studies have highlighted this enzyme as being highly expressed in many solid tumors, including breast cancer, and to be correlated with poor survival. However, the metabolic functions of MTHFD2 in cancer cells have not been well defined. To investigate the function of MTHFD2 in breast cancer cells, we generated and characterised MCF-7 cells with stable suppression of MTHFD2 expression using a combination of cellular assays and metabolic profiling. Loss of MTHFD2 caused MCF7 cells to become glycine auxotrophs, i.e. reliant on exogenous glycine, and more sensitive to exogenous folate depletion. Another prominent metabolic alteration observed as a consequence of MTHFD2 suppression was a more glycolytic phenotype, consistent with widespread modifications of cellular metabolism. Collectively, these data suggest that targeting MTHFD2 activity is likely to influence multiple metabolic pathways in breast cancer and could be combined with a range of anti-metabolite therapies.
\end{abstract}




\section{Introduction}

An established characteristic of cancer cells is a distinct metabolic profile compared to normal cells, defined by the altered uptake of nutrients from the environment and differential usage in metabolic pathways. Cancer cells reprogram metabolism in order to sustain high growth rates and to facilitate survival in nutrient- and oxygen-poor environments. Well-established tumor metabolic phenotypes include the Warburg effect, increased uptake of glutamine to support anaplerosis and maintain TCA cycle intermediates, altered fatty acid synthesis and altered serine and glycine metabolism ${ }^{1-}$

3 . The metabolic reprogramming of cancer cells is necessarily driven by the altered function of metabolic enzymes in tumors, with several metabolic enzymes identified as proto-oncogenes or tumor suppressors. A plethora of mechanisms can result in the altered activity of metabolic enzymes in cancers including the acquisition of mutations that disrupt normal function or deregulated expression driven by oncogenes such as HIF-1 $\alpha$, c-MYC or SREBP $1^{4}$.

Folate is a water-soluble B-vitamin that humans obtain from their diet. Complex metabolic pathways that involve folate and its derivatives generate essential precursors that sustain cell growth and the antioxidative/methylation capacities of proliferating cells (Fig.1). Folate metabolism involved the uptake of folate from the environment, followed by its conversion to tetrahydrofolate (THF). One-carbon units from donors such as methionine, serine, glycine, dimethylglycine and sarcosine are transferred to folate carriers, which subsequently pass them on to drive biosynthetic reactions. Methyl-THF $\left(\mathrm{CH}_{3}\right.$-THF) donates one-carbon for methylation reactions, methylene THF $\left(\mathrm{CH}_{2}-\mathrm{THF}\right)$ is used for the conversion of dUMP to dTMP, and formyl THF (CHO-THF) is used for purine synthesis ${ }^{5}$. A recent important finding has been that the folate pathway is also a major producer of NADPH, a cofactor that powers reductive biosynthesis and redox defences, during the conversion of $\mathrm{CH}_{2}$-THF to $\mathrm{CHO}-\mathrm{THF}^{6}$.

In mammalian cells, folate metabolism is compartmentalised into cytoplasmic and mitochondrial components 5 . It has been estimated through experiments conducted in mouse embryonic fibroblasts (MEF) that approximately $80 \%$ of one-carbon units that flow into the methylation cycle originate from the mitochondrial pathway ${ }^{7}$. Mitochondrial folate metabolism is also a major source of glycine synthesized from 
serine $^{2}$. MTHFD2 is a metabolic enzyme involved in mitochondrial folate one-carbon metabolism, catalyzing the conversion of $\mathrm{CH}_{2}$-THF to CHO-THF (Fig.1). Importantly $\mathrm{CH}_{2}$-THF cannot be transported between mitochondria and the cytoplasm ${ }^{5}$. Consequently, the suppression of MTHFD2 activity could lead to the accumulation of $\mathrm{CH}_{2}$-THF and blockage of the mitochondrial folate metabolism. Homozygous deletion of MTHFD2 in mice is embryonic lethal, indicating essential roles for this enzyme during development ${ }^{8}$. A number of recent studies have highlighted MTHFD2 as having critical functions in human cancers and being a promising therapeutic target. We and others have previously reported that MTHFD2 is under the regulation of two miRNAs that are frequently deregulated in cancer, miR-9 and miR-22, Enzymes of mitochondrial one-carbon metabolism, including MTHFD2, are highly correlated with proliferation across cancer cell lines ${ }^{2}$. In a separate study, investigation of 1,454 metabolic enzymes in 1,981 human tumors found MTHFD2 to be the most consistently over-expressed ${ }^{11}$. This study also found that MTHFD2 was not present in normal proliferating cells, but appeared to be specific to proliferating transformed cells. Other studies specifically implicate MTHFD2 in breast cancer: knockdown of MTHFD2 in breast cancer cell lines has been found to reduce cell viability and increase apoptosis ${ }^{9}$, to impair migration, invasion and metastasis ${ }^{12}$ and to increase the expression of cancer stem cell markers ${ }^{12}$. Recent studies have found a significant association between mRNA expression ${ }^{10,11}$ and protein expression of MTHFD2 in large cohorts of breast cancer patients ${ }^{13}$.

Despite the growing interest in MTHFD2 as a potential therapeutic target, the metabolic functions of this enzyme in cancer cells have not been defined. Recent studies have suggested that the benefit to cancer cells of high MTHFD2 expression may be independent of the metabolic functions of the enzyme. An alternative enzyme, MTHFD2L, has been identified to also be able to catalyse the oxidation of $\mathrm{CH}_{2}-\mathrm{THF}$ and is expressed in adult cells ${ }^{14,15}$. Another recent study has also found that MTHFD2 localized to the nucleus and that over-expression of the enzyme was sufficient to promote proliferation independent of its dehydrogenase activity ${ }^{16}$. Thus the contribution of metabolic regulation to the role of MTHFD2 in cancer remains an open question.

In this study we aimed to investigate the metabolic function of MTHFD2, specifically in breast cancer cells. For this purpose we generated a breast cancer cell line stably transfected with shRNA suppressing MTHFD2 expression and characterised this 
model using a variety of assays including metabolic profiling. Our findings in this study suggest a role for MTHFD2 in the metabolic reprogramming of breast cancer cells, specifically with respect to glycolysis, folate metabolism and glycine biosynthesis.

\section{Materials \& Methods}

\section{Cell culture}

The cell lines examined in this study were obtained from the American Type Culture Collection (ATCC, USA). The cells were maintained at $37^{\circ} \mathrm{C}$ and $5 \% \mathrm{CO}_{2}$ in Minimum Essential Media (MEM) supplemented with 10\% foetal bovine serum (FBS), 1x non-essential amino acids (NEAA) (catalog number 11140-050), 1 x Lglutamine $(2 \mathrm{mM}), 1 \mathrm{x}$ penicillin/streptomycin (Gibco, USA).

\section{Generation of stable transfected MCF-7 lines}

Hush 29mer constructs containing pCMV-shMTHD2 constructs and the transfection reagent Turbofectin 8 were purchased from Origene Technologies (Rockville, MD, USA). MCF-7 cells were transfected with $1 \mu \mathrm{g}$ of the shRNA constructs or the empty vector. The medium was changed 24 hours after transfection and after a further 24 hours cells were subjected to selection in medium containing G418 $(1.0 \mathrm{mg} / \mathrm{ml}$, Sigma-Aldrich). After 3 weeks in a selection medium, surviving clones were screened for MTHFD2 expression by qRT-PCR and immunoblotting.

\section{Metabolic requirement growth studies}

For the folate depletion experiment, cells were cultured in folate-free RPMI-1640 media supplemented with dialyzed FBS (dFBS, Gibco) or in the same media supplemented with $2.3 \mu \mathrm{M}$ folate (Sigma-Aldrich). Cells were plated at a density of 1000 cells/well in 96-well plates and Cell Counting Kit-8 (Sigma-Aldrich) was used to determine the number of viable cells after 7 days based on the manufacturer's instructions. 
For the serine and glycine depletion experiment, cells were cultured for 4 days in MEM media (Invitrogen) with depletion of serine (normally 10mM), glycine (normally $10 \mathrm{mM}$ ) or both serine and glycine. All other NEAA were supplemented at the same concentration as present in MEM NEAA mix (10mM each, Gibco; catalog number 11140050). The number of cells were determined using a Moxi $^{\mathrm{TM}} \mathrm{z}$ cell counter (Orflo Technologies).

\section{Colony Formation}

Anchorage dependent colony formation was performed by plating 1000 cells/well in $30 \times 15 \mathrm{~mm}$ dishes. On day 9, the colonies were fixed using methanol/acetic acid (3:1) and stained using $0.5 \%$ crystal violet. The total number of colonies in each dish was counted using the 10x objective of an inverted microscope (Olympus CK2, Japan).

To examine anchorage independent colony formation cells were plated at a density of 10000 cells/well in semi-solid agar $(0.4 \%)$, plated on top of a layer of solid agar $(0.6 \%)$. The average number of colonies in a random field was counted on day 13 using the 10x objective of an inverted microscope

\section{ATP level measurement}

Cells were plated at a density of 200,000 cells $/ \mathrm{ml}$ in T25 flasks in normal media and after two days ATP levels were determined by luminescence using the ATP Biolumiescence Assay Kit HS II (Roche, Switzerland).

\section{Quantitative Reverse Transcription PCR}

Total RNA was extracted from cells using Trizol (Invitrogen). Reverse transcription was performed using the High capacity cDNA reverse transcription kit (Applied Biosystems). The qRT-PCR was performed using the Taqman Fast advanced master mix and pre-designed taqman probes (Applied Biosystems) in the ABI 7500 RealTime PCR system and relative mRNA levels were calculated using the $\Delta \Delta \mathrm{C}_{\mathrm{T}}$ method. 


\section{Immunoblotting}

Protein was isolated from cells using RIPA buffer (Sigma-Aldrich) and quantified using a BCA assay (Pierce). Monoclonal anti-MTHFD2 (ab56772) was purchased from Abcam and monoclonal anti-beta-Actin (AC-15) antibody was purchased from Sigma-Aldrich. Protein lysates were resolved in 10\% Acrylamide gels by SDS-PAGE electrophoresis and the relative intensities were determined using the ImageJ software.

\section{${ }^{1}$ H NMR Spectroscopy}

The cell culture media of exponentially growing cells was replenished with MEM media supplemented with dFBS and the media was collected after 24 hours. At the same time, cells were harvested and counted using the trypan blue exclusion method. The collected cell culture media was centrifuged to remove any cell debris. From the supernatant $550 \mu \mathrm{l}$ were transferred to a fresh tube and $50 \mu \mathrm{D}_{2} \mathrm{O}$ containing $0.2 \%$ (w/v) 4,4-dimethyl-4-silapentane-1-ammonium trifluoroacetate (DSA) was added. High resolution ${ }^{1} \mathrm{H}$ nuclear magnetic resonance (NMR) spectra were acquired at 14.1 $\mathrm{T}$ (600.29 MHz ${ }^{1} \mathrm{H}$ frequency) using a Bruker AVANCE 600 spectrometer (Bruker Biospin, Rheinstetten, Germany). Spectra were acquired using a Carr-PurcellMeiboom-Gill pulse sequence, and the sum of 128 free induction decays were collected into $64 \mathrm{k}$ data points with a spectral width of 12019.230 and $0.3 \mathrm{~Hz}$ line broadening.

\section{Gas Chromatography-Mass Spectrometry}

Cells were quenched and intracellular metabolites collected by scraping in $750 \mu \mathrm{L}$ of cold (approx. $-70^{\circ} \mathrm{C}$ ) methanol and aqueous metabolites were extracted using a 2:1:3 chloroform:methanol:water extraction method. The aqueous fractions were transferred to silanized GC-MS vials and lyophilized.

Samples for metabolic profiling were derivatised for GC-MS by a two-step methoximation/silylation derivatization procedure. $\left[\mathrm{U}_{-}{ }^{13} \mathrm{C}_{3}\right]$ serine $(20 \mu \mathrm{l}, 1 \mathrm{mM})$, [U${ }^{13} \mathrm{C}_{6}$ ]glucose $(20 \mu \mathrm{l}, 1 \mathrm{mM})$, and myristic acid d27 $(10 \mu \mathrm{l}, 1.5 \mathrm{mg} / \mathrm{mL})$ were added to the samples as internal standards before drying under reduced pressure. The dried 
samples were first methoximated with a solution of $20 \mathrm{mg} / \mathrm{ml}$ methoxyamine hydrochloride $(20 \mu \mathrm{l})$ in anhydrous pyridine at $30^{\circ} \mathrm{C}$ for $90 \mathrm{~min}$. They were then silylated with $\mathrm{N}$-methyl-N-trimethylsilytrifluoroacetamide (MSTFA) with $1 \%$ trimethylchlorosilane (TMCS) $(80 \mu \mathrm{l})$ at $37^{\circ} \mathrm{C}$ for $30 \mathrm{~min}$. Following derivatization, 2-fluorobiphenyl in anhydrous pyridine $(10 \mu \mathrm{l}, 1 \mathrm{mM})$ was added to the samples as an injection standard.

GC-MS analysis was performed on an Agilent 7890 gas chromatograph equipped with a $30 \mathrm{~m}$ DB-5MS capillary column with a $10 \mathrm{~m}$ Duraguard column and connected to an Agilent 5975 MSD (Agilent Technologies UK Ltd.). Samples were injected with an Agilent 7683 autosampler injector into deactivated splitless liners. Oven temperatures and other general settings were adopted from the Agilent Fiehn Library $\operatorname{method}^{17}$. Briefly, separation was carried out with $1 \mathrm{~mL} / \mathrm{min}$ constant flow helium; oven ramp $60{ }^{\circ} \mathrm{C}$ ( $1 \mathrm{~min}$ hold $)$ to 325 at $10{ }^{\circ} \mathrm{C} / \mathrm{min}, 10 \mathrm{~min}$ hold before cool-down, for a total run time of $37.5 \mathrm{~min}$. MS acquisition was carried out with electron impact ionization at $70 \mathrm{eV}$, transfer line temperature of $290^{\circ} \mathrm{C}$, filament source temperature of $230^{\circ} \mathrm{C}$, and quadrupole temperature of $150^{\circ} \mathrm{C}$. The MS scan range was from 50 to 600 $\mathrm{u}$ at a scan rate of $2.66 \mathrm{spectra} / \mathrm{sec}$ with a 5.90 min solvent delay time.

\section{Metabolomic Data Processing}

${ }^{1} \mathrm{H}$ NMR spectra were imported and manipulated in Matlab (Mathworks, Natick, MA, USA) using in-house code for automatic phasing, baseline correction, and referencing chemical shifts to the TSP resonance at $\delta 0$. Metabolites were assigned using Chenomx NMR suite 8.0 (Chenomx Inc., Alberta, Canada) and the Human Metabolome Database. Individual metabolite peaks were integrated and quantified relative to the DSA signal. After peak integration, the intensity for unconditioned media was subtracted, the intensity difference converted to molar change (taking into account proton number) and then the molar change normalised by cell number at the end of the experiment. Metabolite uptake and production were scaled to mean WT dFBS values.

For GC-MS, metabolites were assigned using the Agilent Fiehn Library with the deconvolution program AMDIS. Analysis was carried out based on Retention Index Data calibrated to fatty-acid methyl ester retention indices from a standard mixture. Mass spectra for matched metabolites were then compared to the deconvoluted mass 
spectra to confirm identification. This was followed by analysis using GaVIN as previously described ${ }^{18}$. This involved visual inspection of peak quality (including match to retention time), rejection of metabolites matched by AMDIS in under $80 \%$ of samples, and back-filling of the sample matrix to provide a numerical value for all samples for each metabolite, based on the quantitation ion for each metabolite as taken from the Fiehn library. Calculated integral values obtained from GaVIN were subjected to total area normalization and scaled to mean WT dFBS values.

\section{Results}

\section{Generation of MCF-7 cells with stable depletion of MTHFD2}

We first determined the levels of the MTHFD2 protein in five cancer cell lines from the NCI-60 panel of cancer cell lines. High expression of MTHFD2 was observed in the two estrogen receptor (ER) positive cell lines (MCF-7 and T47D) and one ER negative cell line (HS-578T), but was lower in the remaining two ER negative cells lines (MDA-MB-231 and BT-549, Suppl.Fig.1). In these cell lines, there seems to be no clear association between estrogen receptor status and MTHFD2 protein levels. Subsequently, we proceeded to generate MCF-7 cell lines stably transfected with control non-targeting shRNA (shCON) or an shRNA specific for MTHFD2 (shMTHFD2). The MCF-7 cell line was deemed suitable for this study as it expresses high levels of MTHFD2 (Suppl.Fig.1) and knockdown of MTHFD2 has been reported to affect growth and survival of this line ${ }^{911}$. Knockdown of MTHFD2 was verified through measurements of mRNA and protein levels, with significantly lower levels of MTHFD2 (20\% or less) in the shMTHFD2 cell line compared to the shCON (Fig. 2A,2B). In agreement with previous reports ${ }^{9,11}$, the suppression of MTHFD2 affected MCF-7 cell numbers, with clear effects after 4 days of growth (Fig.2C). No difference was observed in colony formation under adherent conditions in the shMTHFD2 and shCON cells after 72hrs (Suppl. Fig.2A). No statistical difference was observed in colony formation under non-adherent conditions after $72 \mathrm{hrs}$, although a trend towards decreased number of colonies for shMTHD2 cells was observed (Suppl. Fig.2B). No effects were observed on ATP levels between the two cell lines, suggesting that suppression of MTHFD2 did not affect energy production under the conditions tested (Suppl. Fig.2C). 
MTHFD2 suppression renders cells more sensitive to extracellular glycine depletion

In the mitochondria, SHMT2 catalyses the conversion of serine to glycine and generates $\mathrm{CH}_{2}$-THF in the process. The conversion of $\mathrm{CH}_{2}$-THF to CHO-THF (through the action of MTHFD2) and finally formate (through the action of MTHFD1L) allows the recycling of THF, which can be used for other folatedependent reactions (Fig.1). To determine whether MTHFD2 suppression affected the conversion of serine to glycine, the intracellular levels of these amino acids were measured in the shMTHFD2 and shCON MCF-7 cells. An increase in intracellular serine together with a decrease in glycine levels was observed in MCF-7 cells following suppression of MTHFD2, consistent with reduced production of glycine from serine (Fig.3A,3B).

A recent study has suggested that cancer cells rely more on serine than glycine to support growth and metabolism ${ }^{20}$. A similar trend was observed in the five breast cancer cell lines we examined; serine supplementation of basal media enhanced the growth of all the cell lines, glycine supplementation increased growth of only one cell line, while formate did not affect the growth of any of the cell lines examined (Suppl.Fig.3). Nevertheless, disruption of mitochondrial folate metabolism can render cells more reliant on exogenous glycine ${ }^{2,5,21}$.

To examine how suppression of MTHFD2 affected the dependence of breast cancer cells on exogenous serine and glycine, shMTHFD2 and shCON were cultured in media in which serine, glycine, or both serine and glycine were depleted (Fig.3C). Exogenous serine was necessary for the cell growth of MCF-7 cells irrespective of MTHFD2 status. When only glycine was depleted, shCON cells (containing functional MTHFD2) showed no reduction in growth in the presence of exogenous serine. In contrast, shMTHFD2 cells showed decreased proliferation when only glycine was depleted despite the availability of exogenous serine (Fig.3C). These results suggest that in the absence of MTHFD2 activity, MCF-7 cells become dependent on exogenous glycine and can no longer synthesize sufficient glycine de novo. 


\section{MTHFD2 deficient cells are more sensitive to folate depletion and are not rescued by formate supplementation}

In the absence of other enzymes that can substitute for MTHFD2, suppression of MTHFD2 would lead to the accumulation of $\mathrm{CH}_{2}$-THF in the mitochondria, thus decreasing the available THF pool. This could lead to MTHFD2 cells being more sensitive to folate depletion. To examine this possibility we compared the relative growth of shCON and shMTHFD2 cells in folate-depleted media compared to media containing folate $(2.3 \mu \mathrm{M})$ after a period of seven days. This analysis found that MCF7 cells with suppressed MTHFD2 had a much more prominent decrease in the number of viable cells after 7 days in folate-depleted media than control cells (Fig.4).

Subsequently we examined whether supplementation of the media with formate $(2 \mathrm{mM})$, the end product of mitochondrial folate metabolism, could rescue the effect of folate depletion. Our analysis found that formate rescued the cells with wildtype MTHFD2 expression levels, but not the cells with depleted levels of the enzyme (Fig.4).

\section{MTHFD2 suppression renders cells more glycolytic and increases glutamine consumption}

Next the cell culture media of shCON and shMTHFD2 cell lines were examined to determine whether suppression of MTHFD2 had other effects on MCF-7 metabolism beyond serine/glycine and folate metabolism. Examination of the extracellular media of shCON and shMTHFD2 cells by ${ }^{1} \mathrm{H}$ NMR spectroscopy revealed changes in the consumption (Fig.5A) and release (Fig.5B) of multiple metabolites. Compared to the cells with normal MTHFD2, the shMTHFD2 cells had significantly increased consumption of glucose $(36 \%, \mathrm{p}<0.01)$ and glutamine $(45 \%, \mathrm{p}<0.01)$, while displaying significantly reduced consumption of valine $(38 \%, \mathrm{p}<0.01$, Fig.5A). The shMTHFD2 cells also displayed significantly increased production of lactate, pyruvate, alanine and glutamate (Fig.5B). There was a trend towards decreased production of formate, the end product of mitochondrial one-carbon metabolism, from shMTHFD2 cells but this was not statistically significant $(37 \%, \mathrm{p}=0.05)$. Overall the examination of cell culture media indicated that suppression of MTHFD2 in MCF7 cells caused these to become more glycolytic (increased consumption of glucose and 
production of lactate) together with increased glutaminolysis (increased consumption of glutamine and increased production of glutamate) while also exhibiting reduced mitochondrial metabolism (lower consumption of valine and production of formate). To further examine the effects of MTHFD2 suppression on glucose metabolism, we measured intracellular levels of selected glycolytic and TCA metabolites using GCMS. It was found that MTHFD2 suppression led to decreased intracellular levels of glucose-6-phosphate (G-6-P) and lactate, but not of phosphoenolpyruvate (PEP, Fig.6). For TCA metabolites, increased levels of fumarate and malate were detected but citrate, succinate, and $\alpha$-ketoglutarate were unaffected (Suppl.Fig.4).

\section{Discussion}

A greater understanding of the metabolic functions of MTHFD2 in breast cancer cells is currently needed to clarify its role in breast cancer and the potential therapeutic benefits of targeting the enzyme. In this study, we identified that the repression of this enzyme affected multiple metabolic pathways in MCF-7 breast cancer cells.

We also found that suppression of MTHFD2 in MCF-7 cells resulted in altered levels of intracellular serine and glycine (Fig.3), suggesting that the enzyme is required for the functioning of mitochondrial glycine synthesis in these cells. However, further experiments are required to understand the precise contribution of MTHFD2 to this pathway. Notably, shMTHFD2 cells displayed a reliance on exogenous glycine (Fig.3), consistent with previously reported observations that complete knockout of MTHFD2 rendered mouse embryonic fibroblasts cells glycine auxotrophs (i.e. completely dependent on exogenous glycine in order to grow ${ }^{21}$. This study showed for the first time that suppression of MTHFD2 can also produce the same phenotype in human cancer cells. The shMTHFD2 cells examined here could still grow in the absence of glycine (albeit at a slower rate), although it is not clear whether that is due to residual MTHFD2 activity in these cells, the activity of other enzymes such as MTHFD2L, or the presence of alternative metabolic pathways that are active in cancer cells.

A recent analysis of serine/glycine uptake and usage in cancer cells concluded that exogenous serine is essential for the proliferation of cancer cells, but not glycine ${ }^{20}$, and we have found a similar pattern for breast cancer cell lines studied here (Suppl. 
Fig.3). Supplementation of cell culture media with glycine was also unable to rescue cancer cells after RNAi silencing of MTHFD $2^{11}$. The question therefore arises as to why MCF-7 cells with suppressed MTHFD2 are rendered dependent on exogenous glycine. One possibility is that cytoplasmic folate metabolism alone is insufficient to maintain the necessary levels of glycine in MCF-7 cells with suppressed MTHFD2. A second possibility is that glycine is required by the shMTHFD2 cells for the recycling of accumulated $\mathrm{CH}_{2}$-THF within the mitochondria. Since $\mathrm{CH}_{2}-\mathrm{THF}$ is unable to flow between the mitochondria and cytoplasm, the only process by which folate trapped in this form can be recycled and used for essential biochemical processes is through the conversion of glycine to serine (Fig.1). This would be consistent with our observation that the effect of folate depletion on cell growth cannot be rescued by supplementation with formate, the end product of mitochondrial folate metabolism (Fig.4). However, a major limitation for interpreting and clarifying the link between MTHFD2 repression and the sensitivity to folate depletion is that we did not measure folate metabolites directly in this study, something that needs to be addressed in future work.

A second prominent metabolic phenotype observed in the MCF-7 cells as a consequence of MTHFD2 suppression was an increase in their glycolytic nature. Serine is a direct ligand and allosteric activator of $\mathrm{PKM} 2^{22}$, and it was recently reported that suppression of the mitochondrial enzyme SHMT2 upstream of MTHFD2 limits the activity of the PKM2 in glioma cells by decreasing the levels of its metabolic activators ${ }^{23}$. Increased intracellular serine levels due to disruption of mitochondrial glycine synthesis with MTHFD2 suppression may therefore potentially be responsible for the more glycolytic phenotype observed. Another mechanism by which MTHFD2 depletion could affect glucose metabolism in cancer cells is by necessitating a greater diversion of glycolytic intermediates into the pentose phosphate pathway (PPP) for the generation of NADPH. The recent breakthrough paper by Fan et al. $2014^{6}$ reported that folate metabolism, including the mitochondrial branch, is a major source of NADPH, a necessary cofactor for the anabolic synthesis of macromolecules and for protection against oxidative stress ${ }^{24}$. An increased flow of glucose carbon from glucose-6-phosphate to the PPP, the other major NADPH generating pathway, could therefore be a compensatory mechanism. Interestingly a significant decrease in the intracellular levels of glucose-6-phosphate was observed (Fig.6). One hypothesis that can be tested in future studies is whether cancer cells 
respond to repression of MTHFD2 by increasing flow of carbons to the PPP thus necessitating an increase in glycolysis.

The increased uptake of glutamine that was observed for shMTHFD2 cells (Fig.5) could also relate to compensatory metabolic reprogramming after suppression of MTHFD2. Glutamine can act to replenish TCA intermediates through its conversion to oxaloacetate, citrate and $\alpha$-ketoglutarate ${ }^{25}$. Glutaminolysis can also compensate for the reduced production of NADPH by providing a substrate for malic enzyme ${ }^{26}$. The high expression of MTHFD2 in breast cancer, as well as the association between the levels of this enzyme and poor patient prognosis, demonstrate that altered function of this enzyme confers selective advantages to tumors ${ }^{2,10,12,13}$. However, recent data indicate that MTHFD2 also possesses non-metabolic functions ${ }^{16}$. For example, MTHFD2 was shown to translocate to the nucleus and to be able to drive proliferation of cancer cells independently of its enzymatic activities. Here we demonstrate for the first time that MTHFD2 is an important contributor to the metabolic phenotype of breast cancer cells.

The question naturally arises as to whether the effects of MTHFD2 suppression on mitochondrial folate metabolism and glycolysis underlie the selective advantage conferred by MTHFD2 to cancer cells. In the case of mitochondrial folate metabolism, the benefits of heightened activity of this pathway to cancer cells is more clear: increased production of nucleotides for DNA synthesis, polyamine synthesis, substrates for methylation reactions, and maintenance of redox status ${ }^{2,5,17,20,27}$. In our cell model, the MTHFD2 depletion resulted in increased glycolysis. However, this does not necessarily mean that over-expression of MTHFD2 in cancer cells would result in decreased glycolysis. It was recently demonstrated in glioma cells that high levels of SHMT2 under ischaemic conditions were associated with survival through repression of glycolysis and oxygen consumption ${ }^{23}$. Whether MTHFD2 could also play a similar role in regions of limited oxygen availability in tumor tissue in vivo needs to be examined. The increased glycolysis observed could also relate to decreased production of NADPH through the mitochondrial folate metabolic pathway, a molecule that is needed for maintaining anabolic metabolism in proliferating cancer cells.

Although this study has generated interesting and novel results, there are certain limitations that need to be addressed in future studies. First, we examined the effects of MTHFD2 knockdown in only one cell line. Hence, the extent to which our findings 
are relevant in other cancer cell lines is not clear. Future utilization of 3D cell culture and in vivo studies would greatly augment our findings from this study, which were based solely on 2D cell culture work. A more comprehensive examination of the metabolism of the shMTHFD2 cells is also needed in order to elucidate the metabolic rewiring that occurs in response to loss of the enzyme. Methodologies such as shotgun quantitative GC-MS-MS or LC-MS-MS ${ }^{28}$ could prove highly informative in this regard. Metabolic studies could include a more focused investigation of the PPP and nutrient-dependencies of the cancer cells after depletion of MTHFD2. Interference of MTHFD2 using other methodologies e.g. over-expression or knockout using CRISPR-Cas9 would also add further support to our findings.

\section{Conclusions}

Collectively, the data from this study demonstrate for the first time that the MTHFD2 enzyme contributes to the metabolic reprogramming of breast cancer cells. At least in the MCF-7 cells that were studied here, the more recently discovered MTHFD2L is unable to substitute for suppressed MTHFD2. A more detailed understanding of the role of MTHFD2 in vivo for tumor growth and survival could highlight the conditions under which targeting the enzyme would be therapeutically useful. MTHFD2 is a highly promising target due to its apparently selective expression in cancer cells ${ }^{11}$. Modelling of the structure of MTHFD2 suggests that it is a potentially druggable target $^{29}$. Additionally, it has been shown in mice that serum glycine and serine can be depleted by diet manipulation ${ }^{30}$. The combination of diet manipulation to deplete glycine with the targeting of MTHFD2 could then be an effective strategy for targeting cancer cells.

\section{Associated Content}

\section{$\underline{\text { Supplementary Information }}$}

The Supporting Information is available free of charge on the ACS Publications website at DOI: 
Supplementary Figure S-1 to S-4 show (1) expression of MTHFD2 in breast cancer cell lines from NCI60 cancer cell line panel (2) effects of stable MTHFD2 suppression on MCF-7 breast cancer cell lines on colony formation and ATP levels (3) effect of serine, glycine or formate supplementation on breast cancer cell lines (4) changes in relative intracellular levels of TCA metabolites in shControl and shMTHFD2 cells.

Supplementary File 1 shows calculated values and raw integrals for metabolite measurements by NMR (Sheet 1) and GC-MS (Sheet 2). NMR measurements are shown as Raw Data (right), normalized to DSA and cell number (center), and normalized values scaled to mean WT dFBS values (left). Metabolites are identified by chemical shift (in ppm) and their corresponding ChEBI ('Chemical Entities of Biological Interest') ID number (Degtyarenko et al., 2008). GC-MS data are shown as Raw Data (right), normalized to total area (center), and normalized values scaled to mean WT dFBS values (left). Metabolites are identified by their retention time (in minutes), the Quantification Ion (QIon, as defined by the reference library, in this case, FiehnLib) and their corresponding ChEBI ID numbers.

\section{ACKNOWLEDGEMENTS}

HK and CK acknowledge support by the European Community's Seventh Framework Programme-Health (FP7/2007-2013) project DETECTIVE (grant agreement number 266838). SG was supported by a UK Medical Research Council (MRC) Advanced Masters Studentship (training grant MR/J015938/1 awarded to HK/ICL). HK and GV are supported by the EC FP7/2007-2013 project Euro-MOTOR (grant agreement number 259867). CHL is supported by a UK Biotechnology and Biological Sciences Research Council (BBSRC) PhD studentship (grant number BB/F529270/1 for the Institute of Chemical Biology (Imperial College London) Doctoral Training Centre). TY is supported by a UK MRC PhD studentship (Imperial College London Faculty of Medicine, Doctoral Training Award). We would also like to acknowledge help by Dr. Gregory Tredwell, Shyam Solanki, and Kwok Wing Joyce Lam in the conduct of this study.

\section{AUTHOR CONTRIBUTIONS}


CK and HK conceived the project. CK, SG, and HK prepared and wrote the final manuscript and figures with support from other authors. CK generated the shCON and shMTHFD2 cell lines. CK, SG, TY, and GV performed all cell culture experiments and assays. CHL analysed NMR data. HK managed the project. All authors made a significant practical and intellectual contribution to the manuscript.

\section{Conflict of interest}

Authors declare that they have no conflict of interest relating to this study.

\section{References}

(1) Vander Heiden, M. G.; Cantley, L. C.; Thompson, C. B. Science 2009, 324 (5930), 1029-1033.

(2) Jain, M.; Nilsson, R.; Sharma, S.; Madhusudhan, N.; Kitami, T.; Souza, A. L.; Kafri, R.; Kirschner, M. W.; Clish, C. B.; Mootha, V. K. Science 2012, 336 (6084), 1040-1044.

(3) Locasale, J. W. Nature Reviews Cancer 2013, 13 (8), 572-583.

(4) Shaw, R. J.; Cantley, L. C. F1000 Biology Reports 2012, 4, 2.

(5) Tibbetts, A. S.; Appling, D. R. Annual Review ofNutrition. 2010, 30, 57-81.

(6) Fan, J.; Ye, J.; Kamphorst, J. J.; Shlomi, T.; Thompson, C. B.; Rabinowitz, J. D. Nature 2014, 510 (7504), 298-302.

(7) Pike, S. T.; Rajendra, R.; Artzt, K.; Appling, D. R. Journal of Biological Chemistry 2010, 285 (7), 4612-4620.

(8) Di Pietro, E.; Sirois, J.; Tremblay, M. L.; MacKenzie, R. E. Molecular and Cellular Biology. 2002, 22 (12), 4158-4166.

(9) Selcuklu, S. D.; Donoghue, M. T. A.; Rehmet, K.; de Souza Gomes, M.; Fort, A.; Kovvuru, P.; Muniyappa, M. K.; Kerin, M. J.; Enright, A. J.; Spillane, C. Journal of Biological Chemistry 2012, 287 (35), 29516-29528.

(10) Koufaris, C.; Valbuena, G. N.; Pomyen, Y.; Tredwell, G. D.; Nevedomskaya, E.; Lau, C.-H.; Yang, T.; Benito, A.; Ellis, J. K.; Keun, H. C. Oncogene 2015.

(11) Nilsson, R.; Jain, M.; Madhusudhan, N.; Sheppard, N. G.; Strittmatter, L.; Kampf, C.; Huang, J.; Asplund, A.; Mootha, V. K. Nature Communications 2014, 5, 3128.

(12) Lehtinen, L.; Ketola, K.; Mäkelä, R.; Mpindi, J.-P.; Viitala, M.; Kallioniemi, O.; Iljin, K. Oncotarget 2013, 4 (1), 48-63.

(13) Liu, F.; Liu, Y.; He, C.; Tao, L.; He, X.; Song, H.; Zhang, G. Tumour Biol. J. Int. Soc. Oncodevelopmental Biology and Medicine 2014, 35 (9), 8685-8690.

(14) Bolusani, S.; Young, B. A.; Cole, N. A.; Tibbetts, A. S.; Momb, J.; Bryant, J. D.; Solmonson, A.; Appling, D. R. Journal of Biological Chemistry 2011, 286 (7), 
$5166-5174$.

(15) Shin, M.; Bryant, J. D.; Momb, J.; Appling, D. R. Journal of Biological Chemistry 2014, 289 (22), 15507-15517.

(16) Gustafsson Sheppard, N.; Jarl, L.; Mahadessian, D.; Strittmatter, L.; Schmidt, A.; Madhusudan, N.; Tegnér, J.; Lundberg, E. K.; Asplund, A.; Jain, M.; Nilsson, R. Scientific Reports. 2015, 5, 15029.

(17) Kind, T; Wohlgemuth, G; Lee, do Y; Lu, Y; Palazoglu, M,;Shahbaz, S,; Fiehn, O. Analytical Chemistry. $200915 ; 81(24): 10038-48$.

(18) Behrends V, Tredwell GD, Bundy JG. Analytical Biochemistry 2011 $5 ; 415(2): 206-8$.

(19) Fu, T. F.; Rife, J. P.; Schirch, V. Archives of Biochemistry and Biophysics 2001, 393 (1), 42-50.

(20) Labuschagne, C. F.; van den Broek, N. J. F.; Mackay, G. M.; Vousden, K. H.; Maddocks, O. D. K. Cell Reports 2014, 7 (4), 1248-1258.

(21) Patel, H.; Pietro, E. D.; MacKenzie, R. E. Journal of Biological Chemistry 2003, 278 (21), 19436-19441.

(22) Chaneton, B.; Hillmann, P.; Zheng, L.; Martin, A. C. L.; Maddocks, O. D. K.; Chokkathukalam, A.; Coyle, J. E.; Jankevics, A.; Holding, F. P.; Vousden, K. H.; Frezza, C.; O’Reilly, M.; Gottlieb, E. Nature 2012, 491 (7424), 458-462.

(23) Kim, D.; Fiske, B. P.; Birsoy, K.; Freinkman, E.; Kami, K.; Possemato, R. L.; Chudnovsky, Y.; Pacold, M. E.; Chen, W. W.; Cantor, J. R.; Shelton, L. M.; Gui, D. Y.; Kwon, M.; Ramkissoon, S. H.; Ligon, K. L.; Kang, S. W.; Snuderl, M.; Vander Heiden, M. G.; Sabatini, D. M. Nature 2015, 520 (7547), 363-367.

(24) Cairns, R. A.; Harris, I.; McCracken, S.; Mak, T. W. Cold Spring Harbor Symposia on Quantitative Biology 2011, 76, 299-311.

(25) Deberardinis, R. J.; Sayed, N.; Ditsworth, D.; Thompson, C. B. Current Opinion in Genetics \& Development 2008, 18 (1), 54-61.

(26) Wise, D. R.; Thompson, C. B. Trends in Biochemical Sciences 2010, 35 (8), 427-433.

(27) Mehrmohamadi, M.; Liu, X.; Shestov, A. A.; Locasale, J. W. Cell Reports 2014, 9 (4), 1507-1519.

(28) Chen, EI; Yates, JR 3rd. Mol Oncol. 2007 1(2):144-59.

(29) Tedeschi, P. M.; Vazquez, A.; Kerrigan, J. E.; Bertino, J. R. Molecular Cancer Research 2015, 13 (10), 1361-1366.

(30) Maddocks, O. D. K.; Berkers, C. R.; Mason, S. M.; Zheng, L.; Blyth, K.; Gottlieb, E.; Vousden, K. H. Nature 2013, 493 (7433), 542-546.

\section{Figure Legends}

Figure 1. Compartmentalisation of mammalian folate-mediated one-carbon

metabolism. Schematic representation of folate metabolism in animal cells. Folate metabolism is divided into mitochondrial and cytoplasmic compartments. THF: tetrahydrofolate; $\mathrm{CH}_{2}$-THF methylene-tetrahydrofolate; $\mathrm{CH}^{+}$-THF: methynyl- 
tetrahydrofolate; CHO-THF: formyl-tetrahydrofolate. Adomet: S-adenosyl methionine; dTMP: deoxythymidine monophosphate. Adopted from ${ }^{5}$.

Figure 2. Characterization of shMTHFD2 and shCON cell lines (A) qRT-PCR for MTHFD2 in shMTHFD2 or shCON cells.; (B) Representative immunoblot for MTHFD2 in shMTHFD2 or shCON cells; (C) Number of shMTHFD2 and shCON cells counted four days after plating $(n=4)$. Values shown are mean \pm SD. $(* p<0.05$ *** $\mathrm{p}<0.001$ after a Student's t-test).

Figure 3. Effect of MTHFD2 suppression on serine/glycine metabolism and dependence on exogenous glycine. (A) Intracellular serine was quantified by GCMS ( $\mathrm{n}=3)$ and expressed as metabolite levels relative to levels in the shCON line; (B) Intracellular glycine was quantified by GC-MS $(n=3)$ and expressed as metabolite levels relative to levels in the shCON line; (C) Relative cell number of shMTHFD2 and shCON cells were determined using a $\operatorname{Moxi}^{\mathrm{TM}} \mathrm{z}$ cell counter for cultures containing serine $(10 \mu \mathrm{M})$, glycine $(10 \mu \mathrm{M})$, neither or both $(10 \mu \mathrm{M}$ each) after four days in culture $(n=4$ replicates for each condition). Values shown are mean $\pm \operatorname{SD}(*$ $\mathrm{p}<0.05 * * \mathrm{p}<0.01 * * * \mathrm{p}<0.001$ after a Student's t-test).

Figure 4. MTHFD2 depleted cells are more sensitive to folate depletion. The relative number of viable shCON and shMTHFD2 cells after 7 days in folate-free RPMI media with or without formate supplementation $(2 \mathrm{mM})$ compared to cells grown in media containing folate $(2.3 \mu \mathrm{M})$ were determined using the CCK-8 kit. Culture media was changed every two days until day 7. Data are presented as mean \pm SEM of three independent experiments $(* * \mathrm{p}<0.01 * * * \mathrm{p}<0.001$ after a Student's ttest).

Figure 5. Consumption and release of metabolites in cell culture media of ShMTHFD2 and shCON cells. Metabolite changes in the cell culture media were determined by ${ }^{1} \mathrm{H}$ NMR spectroscopy after 24 hours, normalised to total cell number and time of culture and expressed relative to shCON (100\%) (A) Comparison of consumption rate of metabolites from cell culture media by shCON and shMTHFD2 cells; (B) Comparison of production rate of metabolites from cell culture media by 
shCON and shMTHFD2 cells. The data are expressed as means \pm SD of three replicates. $(* * \mathrm{p}<0.01$ after a Student's t-test).

Figure 6. Examination of intracellular glycolytic metabolites from shMTHFD2 and shCON cells. Intracellular metabolites were measured by GC-MS and expressed as metabolite levels relative to levels in the shCON line. Figure shows levels of (A) Glucose-6-phosphate (G-6-P) ; (B) Lactate ; and (C) Phosphoenolpyruvate (PEP). The data are expressed as means \pm SD of four replicates $(* * p<0.01$ after a Student's t-test). 